\title{
Implementation of K-means Clustering on SIPP-KLING Dashboard Applications
}

\author{
Fatona Fadilla Rohma, Iklima Ermis Ismail, Yoyok S Waluyo \\ Program Studi Teknik Informatika \\ Jurusan Teknik Informatika dan Ko mputer \\ Politeknik Negeri Jakarta \\ fatonafadilla@gmail.com, iklimaermis.ismail@tik.pnj.ac.id,yoyok sw@yahoo.com
}

\begin{abstract}
This research focuses on grouping health house (rumah_sehat) data into fi ve clusters, namely Very Unhealthy, Unhealthy, Not Heal thy Yet, Healthy, Very Healthy. There were 17 criteria as input parameters for $\mathrm{K}$-Means calculation. These research aims to grouping 8969 houses into the clusters. The results of these clustering can help decision maker (government) to analyze which parts of the houses whose need the attention more, or which areas that lower than healthy standards. The test result shows that there were 3308 Very Healthy, 2496 Healthy, 792 Not heal thy Yet, 1706 Unhealthy, and 667 Very Unheal thy houses. The accuracy of this method was found 87.05\% using confusion matrix, with precision of $\mathbf{9 5 . 6 4 \%}$ and $75,81 \%$, and recall of $83.82 \%$ and 92 , $98 \%$. Based on ROC the level of diagnostic value accur acy of $87.05 \%$ includes good clustering.
\end{abstract}

Keywords : k-means, healthy_home, clustering, data mining

I.

\section{INTRODUCTION}

SIPP-KLING is an environmental health mapping profile information system for UPT Puskesmas Limo. This application aims to facilitate service, health, coaching and even assistance to the community to implement PHBS (Clean and Healthy Life Behavior) in the surrounding environment. In the current SIPP-KLING system, there were 2 categories of final results, namely Healthy and Unhealthy which was obtained from the final total value. Because it only has 2 categories, the analysis obtained from each village is not specific. So that supervision and evaluation cannot be carried out optimally because the gap in the same region is too wide, hence the houses in the same region (categorie) have different health problems and require different handling. With a lack of analysis it also has an impact on the funds that will be given to each region. The amount of funds channeled will not reach the areas that need it most.

Based on the condition above, there is a need to improve the grouping of the SIPP-KLING datasets to get more specific groups. This research use $\mathrm{K}$ Means Clustering to grouping 8969 houses into the cluster, to extracts the information and important patterns of interest in the SIPP-KLING application. K-Means is a most widely used and well studied method in data mining [1]. Refer to [2], the clustering analysis is useful to draw meaningful information or drawing interesting patterns form data sets and used in many fields like bioinformatics, pattern recognition, image processing, data mining, marketing, economics, etc., to get the hidden knowledge.

\section{K-MEANS CLUS TERING}

Clustering is a process of grouping data objects into disjointed group called clusters, so that the data in the same cluster are similar and different to other cluster [3]. The main aim of clustering is to offer a combination of imilar objects. Between classification and clustering are confuse to be different, but in classification objects is assigned in predefined classes while in clustering classes is created [1].

The K-Means algorithm uses the process repeatedly to get the cluster database. It takes the desired number of initial clusters as input and produces the number of end clusters as output. If the algorith $\mathrm{m}$ is needed to generate cluster $\mathrm{K}$ then there will be an initial $\mathrm{K}$ and a final $\mathrm{K}$. The K-Means method will randomly select the $\mathrm{k}$ pattern as the starting point of the centroid. The number of iterations to reach the centroid cluster will be affected by the prospective random initial centroid cluster where the position of the new centroid does not change. The $\mathrm{K}$ value chosen as the initial center will 
be calculated using the Euclidean Distance formula, which is to find the closest distance between the centroid point and the data / object. Data that has a short distance or closest to the centroid will form a cluster [1].

In its completion, the K-Means algorithm will produce the centroid point which is the purpose of the K-Means algorithm. After the iteration of K-Means stops, each object in the dataset becomes a member of a cluster. The cluster value is determined by looking for all objects to find clusters with the closest distance to the object. The K-means algorithm will group data items in a dataset into a cluster based on the closest distance [4]. Following are the steps of K-means algorith $\mathrm{m}[5]$ :

INPUT : Number of desired clusters K Data Object $\mathrm{D}=\{\mathrm{d} 1, \mathrm{~d} 2, . . \mathrm{dn}\}$

Step:

- randomly elevate $\mathrm{K}$ data objects (as initial centers) from data set $\mathrm{D}$.

- Repeat;

- Calculate the distance of each data object $\mathrm{d}_{\mathrm{i}}(1$ $\leq \mathrm{i} \leq \mathrm{n})$ from all $\mathrm{k}$ clusters $\mathrm{C} \mathrm{j}(1 \leq \mathrm{j} \leq \mathrm{k})$ and then as sign data object $\mathrm{d}_{\mathrm{i}}$ to the nearest cluster.

- For each cluster $\mathrm{j}(1 \leq \mathrm{j} \leq \mathrm{k})$

- Recalculate the cluster center until no change in the center of clusters.

OUTPUT : A set of K clusters

\section{DES IGN AND REALIZATION}

\section{A. Design}

Dashboard SIPP-KLING (Profile Information System Mapping Environmental Health) is a webbased system developed by applying K-means clustering methods in data processing to become information that will be easy to learn knowledge. The actors involved in this application are the admin and cadre, the admin manages the SIPP-KLING web dashboard while the cadres are actors who collect the data through the citizen. This system serves admin to control the activities of data processing, and facilitate the admin in making reports and making decisions.

\section{B. Implementation of K-Means Algorithm}

This stage will explain the steps to operate the K-Means algorithm manually:

1. Determination of the number of clusters is five ( $k$ $=5$ ) to be made, the determination of clusters is based on conceptual observations by experts grouped into five, namely, Healthy, Unhealthy, Unhealthy, Very Healthy, Very Unhealthy. The amount of data used is 8969 SIPP-KLING data and 17 attributes from the criteria for determining the Dinkes Health House. The criteria are taken based on interview process with dr. Tiur. The criteria are initialized by $\mathrm{r}$ ( $\mathrm{r} 1$ up to $\mathrm{r} 17$ ) namely: ceiling, wall, floor, the bed room window, the family room window, ventilation, kitchen exhaust fan, lighting, toilet, safe drinking water, sewer system, rubbish management, opening the bed room window, opening the family room window, keep the house and the yard clean, throw the feces of the baby and toddler into the toilet, and throw the garbage in its place.

2. Determination of the centroid of each cluster can be seen in TABLE 1 .

TABLE 1. The Centroid Value of Clusters

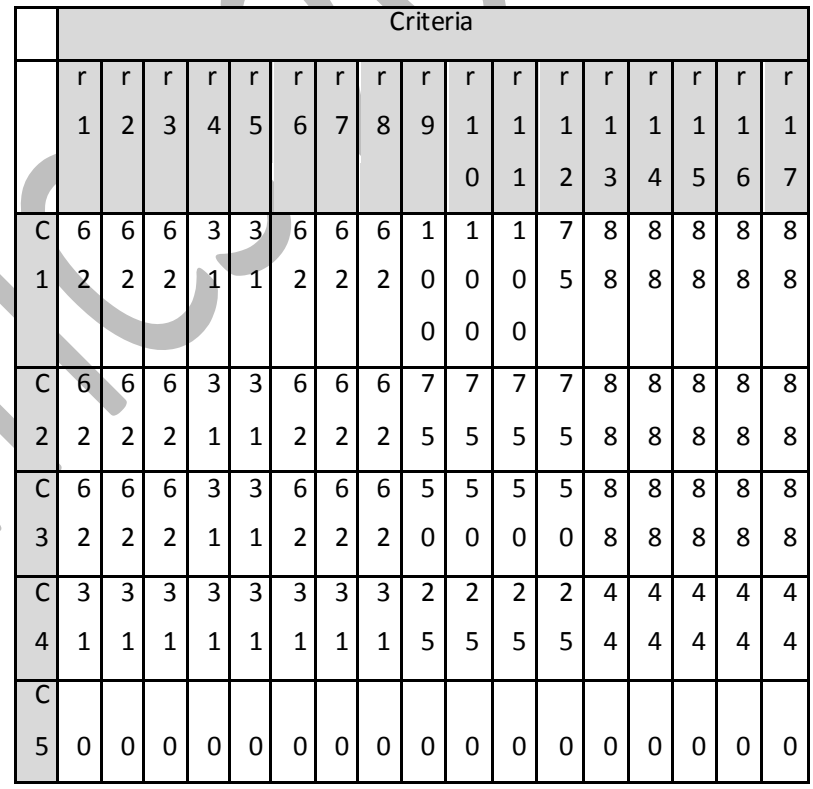

3. Calculate the distance between data and the centroid.

Measuring the distance between the data and the centroid used Euclidian Distance formu la (eq 1)

$d(x, y)=|x-y|=\sqrt{\sum_{i=1}^{n}\left(x_{i}-y_{i}\right)^{2}}$

Then the distance matrix will be obtained, namely $\mathrm{C} 1, \mathrm{C} 2, \mathrm{C} 3, \mathrm{C} 4$ and $\mathrm{C} 5$ as follows:

Data distance of cluster $\mathbf{1}$ is:

- $\quad \mathbf{d}(\mathbf{X 1}, \mathbf{C 1})=\sqrt{ }(62-62)^{2}+(62-62)^{2}+(62-$ $62)^{2}+(0-31)^{2}+(0-31)^{2}+(0-62)^{2}+(31-$ $62)^{2}+(31-62)^{2}+(100-100)^{2}+(100-100)$ ${ }^{2}+(50-100)^{2}+(50-75)^{2}+(0-88)^{2}+$ $(88-88)^{2}+(88-88)^{2}+(88-88)^{2}+(44-88)$ ${ }^{2}=143.153763485$ 
- $\quad \mathbf{d}(\mathbf{X 2}, \mathbf{C 2})=\sqrt{ }(62-62)^{2}+(62-62)^{2}+(62-$ $62)^{2}+(31-31)^{2}+(31-31)^{2}+(31-62)^{2}+$ $(62-62)^{2}+(62-62)^{2}+(100-100)^{2}+(75-$ $100)^{2}+(100-100)^{2}+(75-100)^{2}+(88-88)^{2}$ $+(88-88)^{2}+(88-88)^{2}+(88-88)^{2}+(88-88)$ ${ }^{2}=\mathbf{3 9 . 8 2 4 6 1 5 5 0 3 5}$

- $\quad \mathbf{d}(\mathbf{X 3}, \mathbf{C 3})=\sqrt{ }(62-62)^{2}+(62-62)^{2}+(62-$ $62)^{2}+(0-31)^{2}+(31-31)^{2}+(31-62)^{2}+$ $(31-62)^{2}+(31-62)^{2}+(100-100)^{2}+(100-$ $100)^{2}+(100-100)^{2}+(25-100)^{2}+(0-88)^{2}$ $+(44-88)^{2}+(88-88)^{2}+(88-88)^{2}+(88-$ $88)^{2}=\mathbf{1 2 6 . 5 8 5 9 3 9 1 8 8}$

- $\quad \mathbf{d}(\mathbf{X 4}, \mathbf{C 4})=\sqrt{ }(62-62)^{2}+(62-62)^{2}+(62-$ $62)^{2}+(31-31)^{2}+(31-31)^{2}+(31-62)^{2}+$ $(31-62)^{2}+(62-62)^{2}+(100-100)^{2}+(100-$ $100)^{2}+(100-100)^{2}+(50-100)^{2}+(88-88)$ ${ }^{2}+(88-88)^{2}+(88-88)^{2}+(88-88)^{2}+(88-$ $88)^{2}=\mathbf{5 0 . 4 6 7 8 1 1 5 2 3 8}$

- $\quad \mathbf{d}(\mathbf{X 5}, \mathbf{C 5})=\sqrt{ }(62-62)^{2}+(62-62)^{2}+(31-$ $62)^{2}+(31-31)^{2}+(31-31)^{2}+(31-62)^{2}+$ $(31-62)^{2}+(31-62)^{2}+(100-100)^{2}+(100-$ $100)^{2}+(50-100)^{2}+(50-100)^{2}+(44-88)^{2}$ $+(44-88)^{2}+(88-88)^{2}+(88-88)^{2}+(44-$ $88)^{2}=\mathbf{1 1 3 . 0 3 5 3 9 2 6 8 7}$

The calculation is done to another data distance for clusters 2, 3, 4 and 5 .

4. Then grouping the data according to its cluster, which is the data that has the shortest distance. TABLE 2 shows the result of $1^{\text {st }}$ iteration calculation.

TABLE 2. The result of $1^{\text {st }}$ Iteration

\begin{tabular}{|l|l|l|l|l|}
\hline \multicolumn{5}{|c|}{ Iteration 1 } \\
\hline \multicolumn{1}{|c|}{ C1 } & \multicolumn{1}{c|}{ C2 } & \multicolumn{1}{c|}{ C3 } & \multicolumn{1}{c|}{ C4 } & \multicolumn{1}{c|}{ C5 } \\
\hline 143.153 & 140.953 & 11.484819 & 158.46135 & 252.23401 \\
763485 & 893171 & 124332 & 1754931 & 8324254 \\
\hline 39.8246 & 47.0212 & 84.917607 & 175.31400 & 303.43533 \\
155035 & 71782 & 125967 & 4004244 & 0836737 \\
\hline 126.585 & 133.787 & 147.13599 & 168.70981 & 266.77518 \\
939188 & 144375 & 1518051 & 0028937 & 6252395 \\
\hline 50.4678 & 66.4981 & 97.066987 & 176.13631 & 300.73243 \\
115238 & 202742 & 178958 & 0850432 & 9221312 \\
\hline 113.035 & 110.236 & 121.04544 & 135.25531 & 244.43813 \\
392687 & 110236 & 6010992 & 4128503 & 1231606 \\
\hline \multicolumn{5}{|c|}{} \\
\hline
\end{tabular}

5. The process returns again step 2 .

For the next repetition (1st repetition to completion), the new centroid is calculated by calculating the average value of the data in each cluster, like shown in TABLE 3.
TABLE 3. Average Centroid Value in 1st Repeat

\begin{tabular}{|l|l|l|l|l|l|l|l|l|l|l|l|l|l|l|l|l|l|}
\hline & \multicolumn{10}{|c|}{ The Criteria } \\
\hline & $\mathrm{r}$ & $\mathrm{r}$ & $\mathrm{r}$ & $\mathrm{r}$ & $\mathrm{r}$ & $\mathrm{r}$ & $\mathrm{r}$ & $\mathrm{r}$ & $\mathrm{r}$ & $\mathrm{r}$ & $\mathrm{r}$ & $\mathrm{r}$ & $\mathrm{r}$ & $\mathrm{r}$ & $\mathrm{r}$ & $\mathrm{r}$ & $\mathrm{r}$ \\
& 1 & 2 & 3 & 4 & 5 & 6 & 7 & 8 & 9 & 1 & 1 & 1 & 1 & 1 & 1 & 1 & 1 \\
& & & & & & & & & & 0 & 1 & 2 & 3 & 4 & 5 & 6 & 7 \\
\hline $\mathrm{C}$ & 5 & 6 & 6 & 2 & 3 & 5 & 4 & 5 & 9 & 9 & 9 & 6 & 6 & 6 & 8 & 7 & 8 \\
1 & 9 & 1 & 1 & 7 & 0 & 4 & 9 & 7 & 9 & 1 & 7 & 1 & 5 & 7 & 5 & 9 & 1 \\
&. &. &. &. &. &. &. &. &. &. &. &. &. &. &. &. &. \\
& 8 & 4 & 7 & 7 & 5 & 0 & 5 & 7 & 0 & 8 & 5 & 0 & 7 & 4 & 9 & 8 & 9 \\
& 7 & 8 & 0 & 5 & 4 & 5 & 7 & 7 & 3 & 6 & 7 & 3 & 8 & 3 & 7 & 0 & 2 \\
\hline $\mathrm{C}$ & 3 & 5 & 5 & 5 & 9 & 9 & 5 & 6 & 6 & 6 & 8 & 8 & 7 & 3 & 5 & 5 & 5 \\
2 & 0 & 4 & 1 & 9 & 7 & 6 & 0 & 0 & 6 & 6 & 6 & 4 & 6 & 0 & 4 & 1 & 9 \\
&. &. &. &. &. &. &. &. &. &. &. &. &. & &. &. &. \\
& 1 & 5 & 2 & 3 & 1 & 5 & 7 & 1 & 4 & 8 & 7 & 0 & 9 & 1 & 5 & 2 & 3 \\
& 7 & 5 & 9 & 8 & 3 & 4 & 1 & 6 & 9 & 9 & 8 & 9 & 6 & 7 & 5 & 9 & 8 \\
\hline $\mathrm{C}$ & 3 & 5 & 4 & 5 & 8 & 8 & 4 & 1 & 5 & 6 & 8 & 8 & 6 & 3 & 5 & 4 & 5 \\
3 & 0 & 4 & 7 & 5 & 7 & 4 & 7 & 6 & 5 & 0 & 6 & 1 & 0 & 0 & 4 & 7 & 5 \\
&. &. &. &. &. &. &. &. &. &. &. &. &. &. &. &. &. \\
& 7 & 5 & 9 & 8 & 2 & 7 & 6 & 2 & 7 & 3 & 8 & 2 & 4 & 7 & 5 & 9 & 8 \\
& 2 & 0 & 5 & 7 & 1 & 4 & 8 & 3 & 5 & 1 & 3 & 6 & 8 & 2 & 0 & 5 & 7 \\
\hline $\mathrm{C}$ & 2 & & & & & & & & & & & & & & & & \\
4 & 6 & 3 & 2 & 4 & 7 & 7 & 5 & 2 & 2 & 3 & 7 & 4 & 2 & 2 & 3 & 2 & 4 \\
&. & 2 & 7 & 5 & 4 & 4 & 0 & 9 & 6 & 3 & 7 & 5 & 8 & 6 & 2 & 7 & 5 \\
& 4 &. &. &. &. &. &. &. &. &. &. &. &. &. &. &. &. \\
& 9 & 0 & 0 & 2 & 3 & 5 & 3 & 0 & 1 & 2 & 2 & 3 & 8 & 4 & 0 & 0 & 2 \\
& 5 & 0 & 7 & 3 & 3 & 6 & 7 & 9 & 4 & 5 & 0 & 8 & 5 & 9 & 0 & 7 & 3 \\
\hline $\mathrm{C}$ & & & & & & & & & & & & 1 & 2 & & & & \\
5 & & & & & & & & & & & & 7 & 6 & & & & \\
& & & & & 3 & 3 & 3 & & & & 4 &. &. & & & & \\
& 0 & 0 & 0 & 0 & 0 & 0 & 0 & 5 & 0 & 0 & 4 & 6 & 4 & 0 & 0 & 0 & 0 \\
\hline
\end{tabular}

If the new centroid is different from the previous centroid, then the process continues the next step. However, if the new centroid is calculated the same as the previous centroid, then the clustering process is complete.

In this calculation, the processes stop at $12^{\text {th }}$ iteration, like shown in TABLE 4 and TABLE 5. After getting the cluster label for each data, the average value is searched by adding up all me mbers of each cluster and dividing the number of members.

TABLE 4. Data Grouping in the 12th Repetition

\begin{tabular}{|l|l|l|l|l|}
\hline \multicolumn{5}{|c|}{ Iteration 11 } \\
\hline \multicolumn{1}{|c|}{ C1 } & \multicolumn{1}{c|}{ C2 } & \multicolumn{1}{c|}{ C4 } & \multicolumn{1}{c|}{ C5 } \\
\hline 131.9010 & 120.9553 & 114.7087 & 85.13399 & 123.3096 \\
7031862 & 2084984 & 2330186 & 6868447 & 3521650 \\
50 & 30 & 70 & 0 & 50 \\
\hline 39.07710 & 70.46932 & 112.7033 & 109.2693 & 138.9591 \\
5049157 & 1117856 & 5871371 & 1585632 & 6512706 \\
2 & 9 & 70 & 40 & 30 \\
\hline 108.2886 & 114.6481 & 133.8441 & 47.83863 & 126.9050 \\
0321233 & 4719265 & 1196885 & 9555203 & 5074306 \\
00 & 70 & 80 & 3 & 00 \\
\hline 44.57767 & 70.20964 & 102.7415 & 98.55140 & 134.6284 \\
4052077 & 2990202 & 7594922 & 1356583 & 6808694 \\
6 & 1 & 60 & 8 & 00 \\
\hline 97.92594 & 82.62801 & 77.29028 & 65.02538 & 97.25940 \\
2945531 & 1956220 & 7574655 & 2327006 & 5221802 \\
5 & 2 & 2 & 4 & 3 \\
\hline
\end{tabular}


TABLE 5. Centroid Average Value in 12th Repetition

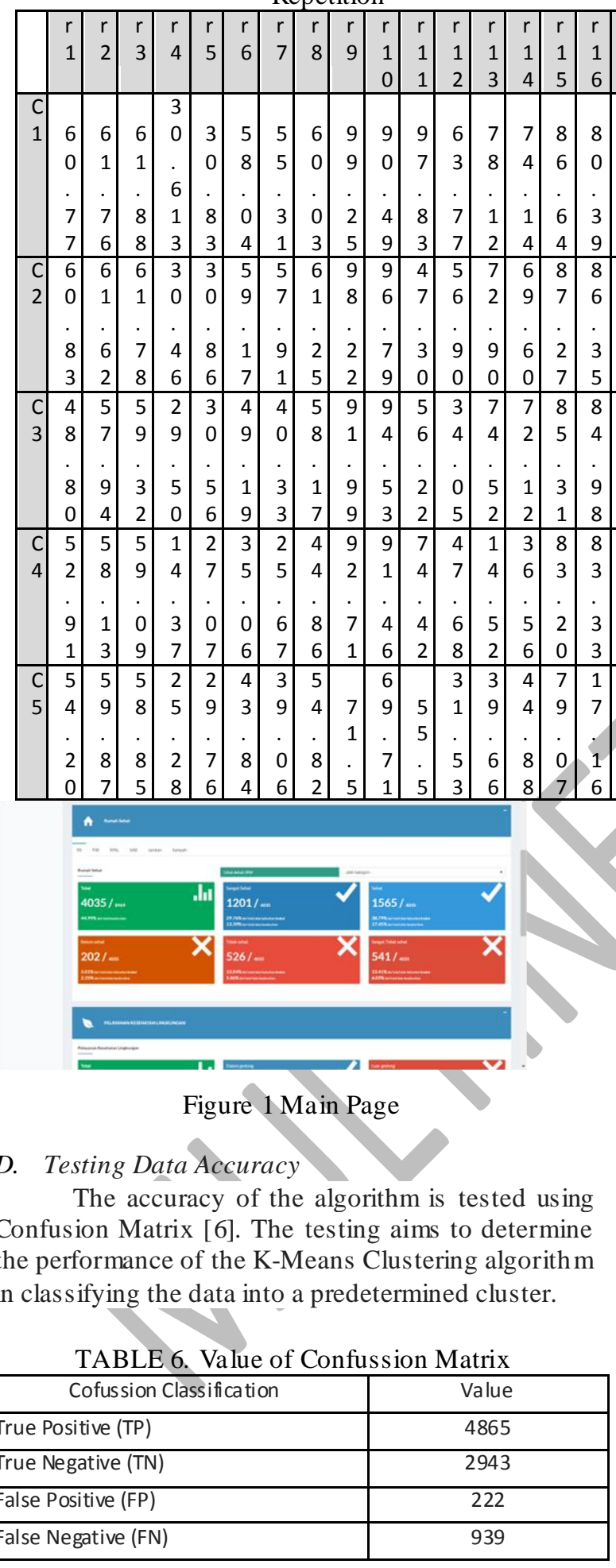

a. Precision
Because the centroid doesn't change (same as the previous centroid), the clustering process is complete, then the data will be grouped based on the closest distance to the cluster, from 8969 K-Means data managed to group 3308 into Very Healthy categories, 2496 Healthy categories, 792 Unhealthy categories, 1706 Unhealthy categories, and 667 Very Unhealthy categories.

\section{System Implementation}

Figure 1 is the implementation of the main page who displays the SIPP-KLING dashboard. On this page the system displays the total amount of data based on the status category such as whether the place is healthy or not, feasible or not, and many or few deviations. The grouped data is the result of the KMeans process. Figure 2 is an implementation of the analysis page for each village, analysis of the location is carried out in each attribute to see which attributes most influence the village. The highest scale on the graph is the scale that most influences and becomes a warning for a village. On the page there are several buttons, the first button to send the status of the database to the database, the second button to send the distance value of the attribute to the database.

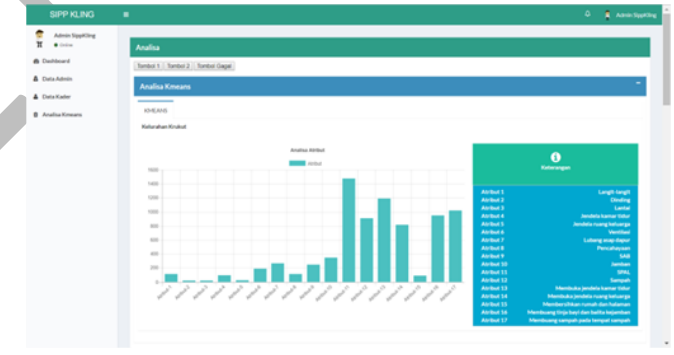

Figure 2 Analys is Page

Precision is the amount of data that is true positive (the amount of positive data that is correctly recognized as positive) is divided by the amount of data that is recognized positively. From the test

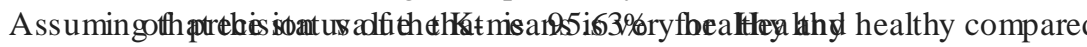
House and $75.71 \%$ for Unhealthy House.

TP / FP + TP) x 100\% $\leftrightarrow 4865 /(4865+222)$ $=0.956359$ (Healthy House)

TN / $(\mathbf{F N}+\mathbf{T N}) \times \mathbf{1 0 0} \% \leftrightarrow 2943 /(2943+939)$ $=0.7571$ (Unhealthy House)

b. Recall

Recall is the amount of data which is true positive divided by the amount of data which is actually positive (true positive + true negative). For recall 
value is $83.82 \%$ for Healthy House and $92.98 \%$ for Unhealthy House.

TP / (FN + TP) x 100\% ↔ $4865 /(939+4865)$ $=0.8382$ (Healthy House)

TN / $(\mathbf{F P}+\mathbf{T N}) \times \mathbf{1 0 0} \% \leftrightarrow 2943 /(222+2943)$ $=0.9298$ (Unhealthy House)

c. Accuracy

By knowing the amount of data grouped correctly it can be seen the accuracy of the prediction that is:

$(\mathbf{T P}+\mathbf{T N}) /(\mathbf{T P}+\mathbf{T N}+\mathbf{F P}+\mathbf{F N}) \times 100 \% \leftrightarrow$ $(4865+2943) /(4865+939+222+2943)=$ 0.8705541309

Refer to [7], the accuracy value is 0.870 , and include as good classification.

\section{d. F-Measure}

F-Measure is a value obtained from precision and recall measurements between clustered classes and actual classes. The ratio of f-measure is reversed, so the higher the fmeasure value, the smaller the difference between the same recall precision.

F-Measure $=2 /(1 /$ recall $+1 /$ precision $)$ or $\mathbf{F}(\mathbf{i}, \mathbf{j})=(2 * \operatorname{recall}(\mathbf{i}, \mathbf{j}) * \operatorname{precision}(\mathbf{i}, \mathbf{j})) /$ $((\operatorname{precision}(\mathbf{i}, \mathbf{j})+\operatorname{recall}(\mathbf{i}, \mathbf{j}))$

For Healthy House :

$2 /(1 / 0.9563+1 / 0.8382)=0.893363789$

$(2 * 0.9563 * 0.8382) /(0.9563+0.8382)=$ 0.893363789

For Unhealthy House :

$2 /(1 / 0.7581+1 / 0.9298)=0.835216992$

$(2 * 0.7581 * 0.9298) /(0.7581+0.9298)=$ 0.83521

e. Data Analysis

From the above test results shows the accuracy of this method is $87,05 \%$, with precision of $95.64 \%$ for healthy class and $75,81 \%$ for unhealthy class, and recall $83.82 \%$ for healthy and $92,98 \%$ to unhealthy. Results accuracy, precision and recall in the grouping of data Healthy House can be seen in the TABLE 7.

TABLE 7 Accuracy Result

\begin{tabular}{|l|c|c|c|}
\hline $\begin{array}{l}\text { Accuracy } \\
87,05 \%\end{array}$ & $\begin{array}{c}\text { True } \\
\text { Healthy }\end{array}$ & $\begin{array}{c}\text { True } \\
\text { Unhealthy }\end{array}$ & $\begin{array}{c}\text { Class } \\
\text { Precision }\end{array}$ \\
\hline Pred. Healthy & 4865 & 939 & $95,64 \%$ \\
\hline Pred. Unhealthy & 222 & 2943 & $75,81 \%$ \\
\hline Class Recall & $83,82 \%$ & $92,98 \%$ & \\
\hline
\end{tabular}

